\title{
Strong convergence of the split-step $\theta$-method for stochastic age-dependent capital system with Poisson jumps and fractional Brownian motion
}

\author{
Ting Kang ${ }^{1,2}$ and Qimin Zhang ${ }^{1 *}$
}

\section{"Correspondence: \\ qiminzhang89@sina.com \\ 1 School of Mathematics and \\ Statistics, Ningxia University, \\ Yinchuan, P.R. China \\ Full list of author information is \\ available at the end of the article}

\section{空 Springer}

\begin{abstract}
Most stochastic age-dependent capital systems cannot be solved explicitly, so it is necessary to develop numerical methods and study the properties of numerical solutions. In this paper, we consider a class of stochastic age-dependent capital systems with Poisson jumps and fractional Brownian motion ( $\mathrm{fBm}$ ) and investigate the convergence of the split-step $\theta$-method ( $\mathrm{SS} \theta$ ) for this system. It is proved that the numerical approximation solutions converge to the analytic solutions for the equations, and the order of approximation is also provided. Finally, a numerical experiment is simulated to illustrate that the $\mathrm{SS} \theta$ method has better accuracy than the Euler method.
\end{abstract}

Keywords: Stochastic age-dependent capital system; Poisson jumps; Fractional Brownian motion; Split-step $\theta$-method; Strong convergence

\section{Introduction}

For the past few years, stochastic age-dependent capital systems have become increasingly important mathematical tools to portray many financial phenomena in the real world. However, most stochastic age-dependent capital systems cannot be solved explicitly, so it is necessary to develop numerical methods and study the properties of numerical solutions. Recently, the study of numerical methods for the stochastic age-dependent capital system has received a great deal of attention. For example, Zhang et al. [1] discussed the exponential stability of Euler approximation for the stochastic age-dependent capital system with Poisson jumps, and further studied the convergence of Euler method for a class of stochastic age-dependent capital systems with random jump magnitudes and Markovian switching [2, 3]. Subsequently, Zhang et al. [4] constructed a split-step backward Euler (SSBE) method for stochastic age-dependent capital system with Markovian switching, and proved that the SSBE method converges with strong order of one half to the exact solution under the given conditions. In the above literature works, uncertainties in the financial market (i.e., randomness of the external environment) were considered in the form of a standard Brownian motion because of the imperfection of financial market itself, including innovations in technique, introduction of new products, natural disasters, and changes

(c) The Author(s) 2018. This article is distributed under the terms of the Creative Commons Attribution 4.0 International License (http://creativecommons.org/licenses/by/4.0/), which permits unrestricted use, distribution, and reproduction in any medium, provided you give appropriate credit to the original author(s) and the source, provide a link to the Creative Commons license, and indicate if changes were made. 
in laws or government policies. However, the randomness of the external environment is not always well modeled by the standard Brownian motion because of the long-range dependence of price of the financial products. In recent years, some researchers adopted fractional Brownian motion $(\mathrm{fBm})$ to describe this long-range dependence of price in the financial market [5-9]. Therefore, it is a very interesting topic to take Poisson jumps and $\mathrm{fBm}$ into account for the stochastic capital systems, and we will consider the following system in this paper:

$$
\left\{\begin{array}{rlrl}
\frac{\partial K(a, t)}{\partial t}= & {\left[-\frac{\partial K_{t^{-}}}{\partial a}-\mu(a, t) K_{t^{-}}+f\left(t, K_{t^{-}}\right)\right] d t+g\left(t, K_{t^{-}}\right) d B^{H}(t)} & & \\
& +h\left(t, K_{t^{-}}\right) d N(t), & & \text { in } D, \\
K(0, t)= & \varphi(t)=\gamma(t) A(t) F\left(L(t), \int_{0}^{A} K(a, t) d a\right), & & \text { in } t \in[0, T], \\
K(a, 0)=K_{0}(a), & & \text { in } a \in[0, A], \\
N(t)=\int_{0}^{A} K(a, t) d a, & & \text { in } t \in[0, T],
\end{array}\right.
$$

where $K_{t^{-}}=\lim _{s \rightarrow t^{-}} K(a, s), \frac{\partial K_{t^{-}}}{\partial a}=\lim _{s \rightarrow t^{-}} \frac{\partial K_{s}}{\partial a}, D=(0, A) \times(0, T) . K(a, t)$ is the stock of capital goods of age a at time $t$, the total output produced at time $t$ is denoted by $N(t), a$ is the age of the capital, the investment $\varphi(t)$ is a new capital, and $f(t, K(a, t))$ in the capital of age $a$ are the endogenous variables. The maximum physical lifetime of the capital is defined as $A$, the planning interval of calendar time $[0, T]$, the depreciation rate $\mu(a, t)$ of the capital, and the capital density $K_{0}(a)$ (the initial distribution of the capital over age) are given. $\gamma(t) \in(0,1)$ and $A(t)$ denote the accumulative rate and the technical progress at the moment of t, respectively. $F(L(t), N(t))$ is the production function and $L(t)$ is the labor force. $f\left(t, K_{t^{-}}\right) d t+g\left(t, K_{t^{-}}\right) d B^{H}(t)+h\left(t, K_{t^{-}}\right) d N(t)$ denotes effects of the external environment for system (1) which depends on $t$ and $K(a, t) . B^{H}(t)$ is a fractional Brownian motion with Hurst parameter $H \in\left(\frac{1}{2}, 1\right), N(t)$ is a scalar Poisson process with intensity $\lambda>0$, and we assume that $B^{H}(t)$ is independent of $N(t)$.

Up to now, the research on numerical solution of model (1) has been mainly focused on Euler method and backward Euler (BE) method. However, these two methods lack flexibility when they are applied to a stochastic age-dependent capital system. Fortunately, a split-step $\theta$-method (SS $\theta$ method) constructed by Ding et al. [10] for solving nonlinear non-autonomous stochastic differential equations (SDEs) can make up for this shortcoming for the first time. Recently, the SS $\theta$ method has attracted many scholars' attention because of its advantages in dealing with the flexibility and the stability for the SDEs [11-18]. Researchers find that SS $\theta$ method or its improved forms have pretty stable properties, convergence rates, and structure-preserving properties [14, 15, 18-20], and the SS $\theta$ method includes Euler method and split-step backward Euler (SSBE) method by fixing $\theta=0$ and $\theta=1$, respectively. However, as far as we know, few results on the convergence of SS $\theta$ method for stochastic age-dependent capital models with Poisson jumps and fBm have been reported. So, we will devote our main attention to the investigation of the following problems of system (1) in this paper.

(Q1) Will the numerical solution converge to the exact solution when we apply the SS $\theta$ method to the stochastic age-dependent capital models with Poisson jumps and $\mathrm{fBm}(1)$ ?

(Q2) Will convergence accuracy be better than previous numerical methods? 
To answer these questions, the outline of this paper is organized as follows. In Sect. 2, we introduce some basic preliminaries which are essential for our investigation, and the splitstep $\theta$-method for stochastic age-dependent capital system with Poisson jumps and fBm is constructed. In Sect. 3, several lemmas which are useful for our main result are proved. Then, in Sect. 4, we establish the main results that the numerical solution converges to the analytical solution for system (1) in the mean square sense. In Sect. 5, a numerical experiment is carried out to support our theoretical results. Finally, a brief conclusion and our future works are presented in Sect. 6.

\section{Preliminaries and the split-step $\theta$-method}

\subsection{Preliminaries}

In this section, we introduce some necessary definitions and assumptions needed for the subsequent discussions. Throughout this paper, we denote by $L^{2}([0, A])$ the space of functions that are square-integrable over the domain $[0, A]$. Let

$$
V=H^{1}([0, A]) \equiv\left\{\varphi \mid \varphi \in L^{2}([0, A]), \frac{\partial \varphi}{\partial a} \in L^{2}([0, A])\right\},
$$

where $\frac{\partial \varphi}{\partial a}$ is generalized partial derivatives with respect to age $a, V$ is a Sobolev space, $W=L^{2}([0, A])$ such that $V \hookrightarrow W \equiv W^{\prime} \hookrightarrow V^{\prime} . V^{\prime}=W^{-1}([0, A])$ is the dual space of $V$. We denote by $\|\cdot\|,|\cdot|$, and $\|\cdot\|_{*}$ the norms in $V, W$, and $V^{\prime}$ respectively; by $(\cdot, \cdot)$ the scalar product in $W$, and by $\langle\cdot, \cdot\rangle$ the duality product between $V$ and $V^{\prime}$, defined by

$$
\langle\cdot, \cdot\rangle=\int_{0}^{A} u \cdot v d a, \quad u \in V, v \in V^{\prime} .
$$

$K$ is a real separable Hilbert space. For an operator $B \in \mathcal{L}(K, W)$ is the space of all bounded linear operators from $K$ into $W$. We denote by $\|B\|_{2}$ the Hilbert-Schmidt norm, i.e., $\|B\|_{2}^{2}=$ $\operatorname{tr}\left(B W B^{T}\right)$. Let $(\Omega, \mathcal{F}, P)$ be a complete probability space with filtrations $\left\{\mathcal{F}_{t}\right\}_{t \geq 0}$ satisfying the usual conditions (i.e., it is increasing and right continuous, and $\mathcal{F}_{0}$ contains all $P$-null sets).

Let $C=C([0, T] ; W)$ be the space of all continuous functions from $[0, T]$ into $W$ with sup-norm $\|\varphi\|_{C}=\sup _{0 \leq s \leq T}|\varphi(s)|, L_{V}^{P}=L^{P}([0, T] ; V)$, and $L_{W}^{P}=L^{P}([0, T] ; W)$.

Definition 2.1 A fractional Brownian motion (fBm) $B^{H}=\left\{B^{H}(t): t \in R\right\}$ for Hurst parameter $H \in(0,1)$ is a continuous and centered Gaussian process with covariance function

$$
R_{H}(t, s)=\mathbb{E}\left[B^{H}(t) B^{H}(s)\right]=\frac{1}{2}\left(|t|^{2 H}+|s|^{2 H}-|t-s|^{2 H}\right), \quad t, s \in R .
$$

The $\mathrm{fBm}$ is then a standard Brownian motion when $H=\frac{1}{2}$.

Remark 2.2 By Definition 2.1, we obtain that a $\mathrm{fBm} B^{H}(t)$ has the following properties:

(i) $B^{H}(0)=0$ and $\mathbb{E}\left[B^{H}(t)\right]=0$ for all $t \geq 0$;

(ii) $B^{H}(t)$ has homogeneous increments, i.e., $B^{H}(t+s)-B^{H}(s)$ has the same law of $B^{H}(t)$ for all $t, s \geq 0$;

(iii) $B^{H}(t)$ is a Gaussian process and $\mathbb{E}\left[B^{H}(t)\right]^{2}=t^{2 H}, t \geq 0$, for all $H \in(0,1)$;

(iv) $B^{H}(t)$ has continuous trajectories;

(v) For any $\alpha>0$, every $s, t \in R$, we have $\mathbb{E}\left[\left|B^{H}(t)-B^{H}(s)\right|^{\alpha}\right]=\mathbb{E}\left[\left|B^{H}(1)\right|^{\alpha}\right]|t-s|^{\alpha H}$. 
In order to analyze the stochastic age-dependent capital system with Poisson jumps and $\mathrm{fBm}(1)$, we impose the following standard hypotheses:

(A1) $f(t, 0)=0, g(t, 0)=0, h(t, 0)=0, t \in[0, T]$;

(A2) (The Lipschitz condition) There exists a positive constant $l$ for all $x, y \in W$, and $\forall t$, such that

$$
|f(t, y)-f(t, x)| \vee\|g(t, y)-g(t, x)\|_{2} \vee|h(t, y)-h(t, x)| \leq l|y-x|
$$

(A3) $\mu(a, t)$ is a nonnegative measurable function in $D, \gamma(t)$ and $A(t)$ are nonnegative continuous functions in $[0, T]$ such that

$$
\begin{cases}0 \leq \mu_{0} \leq \mu(a, t) \leq \bar{\mu}<\infty, & \mu_{0} \text { and } \bar{\mu} \text { are nonnegative constants; } \\ 0<\gamma(t) A(t) \leq \eta, & \eta \text { is a nonnegative constant in }[0, T]\end{cases}
$$

$$
\left\{\begin{array}{l}
F(L, N) \geq 0 \quad(F(L, 0)=0), \frac{\partial F(L, N)}{\partial L}>0 \\
0<\frac{\partial F(L, N)}{\partial N} \leq \delta, \quad \text { where } \delta \text { is a positive constant. }
\end{array}\right.
$$

Now, we give the theorem concerning the existence and uniqueness of solution for system (1), which is essential to discussing the numerical solution of our system.

Theorem 2.3 Under assumptions (A1)-(A4), system (1) has a unique continuous solution $K(a, t)$ on $D$.

The proof of this theorem is similar to that in Zhang et al. [21].

\subsection{The split-step $\theta$-method}

In this subsection, we present the SS $\theta$ method, and give the discrete scheme for system (1). Let $\tau_{j}$ denote the $j$ th jump of occurrence time $N(t)$. For example, suppose that jumps arrive at distinct, ordered times $\tau_{1}<\tau_{2}<\cdots$, let $0, t_{1}, t_{2}, \ldots, t_{m}$ be the deterministic grid points of $[0, T]$. We will construct approximate solution to (1) at a discrete set of times $\tau_{n}(n=$ $1,2, \ldots)$. This set is the superposition of the random jump times of the Poisson process in $[0, T]$ and satisfies max $\left|\tau_{i+1}-\tau_{i}\right|<\Delta_{t}$ (for the sake of simplicity, we denote $\Delta_{t}$ as $\Delta$ ). Obviously, the random Poisson jump times can be computed without any knowledge of the realized path of (1). Let $\Delta=t_{n+1}-t_{n}, \Delta B_{n}^{H}=B^{H}\left(t_{n+1}\right)-B^{H}\left(t_{n}\right), \Delta N_{n}=N\left(t_{n+1}\right)-N\left(t_{n}\right)$ denote the increment of the time, $\mathrm{fBm}$, and Poisson process respectively. For system (1), the $\operatorname{SS} \theta$ method is defined by the iterative scheme for the time increment $\Delta=\frac{T}{m} \ll 1$,

$$
\begin{aligned}
Q_{t}^{n *}= & Q_{t}^{n}-\frac{\partial Q_{t}^{n+1}}{\partial a} \Delta+(1-\theta)\left(-\mu\left(a, t_{n}\right) Q_{t}^{n}+f\left(t_{n}, Q_{t}^{n}\right)\right) \Delta \\
& +\theta\left(-\mu\left(a, t_{n}\right) Q_{t}^{n *}+f\left(t_{n}, Q_{t}^{n *}\right)\right) \Delta, \\
Q_{t}^{n+1}= & Q_{t}^{n *}+g\left(t_{n}, Q_{t}^{n *}\right) \Delta B_{n}^{H}+h\left(t_{n}, Q_{t}^{n *}\right) \Delta N_{n},
\end{aligned}
$$

with initial values $Q_{t}^{0}=K(a, 0)=K_{0}, Q_{t}^{n}$ is the numerical approximation of $K\left(a, t_{n}\right)$ with $t_{n}=n \Delta, n=0,1,2, \ldots$. Because when $\theta=0$, the SS $\theta$ method becomes the explicit method, and when $\theta=1$, the $\operatorname{SS} \theta$ method is the SSBE method, so we let $\theta \in(0,1)$. 
We will state and prove the following result which is useful for the main result of this paper.

Lemma 2.4 Let assumptions (A2) and (A3) hold, when $0<\Delta<\frac{1}{\theta(l+\bar{\mu})}$, system (1) can be solved uniquely for $Q_{t}^{n *}$ with probability one.

Proof Let $\Phi\left(Q_{t}^{n *}\right)=y+\theta \Delta\left[-\mu\left(a, t_{n}\right) Q_{t}^{n *}+f\left(t_{n}, Q_{t}^{n *}\right)\right], y \in L_{W}^{2}$, and use assumptions (A2) and (A3), we can derive directly from (2) that

$$
\begin{aligned}
|\Phi(u)-\Phi(v)| & \leq \theta \Delta[|f(t, u)-f(t, v)|+|\mu(a, t)(u-v)|] \\
& \leq \theta \Delta(l+\bar{\mu})|u-v|, \quad \forall u, v \in W .
\end{aligned}
$$

Then we can obtain the result via the contraction mapping theorem [22].

Following Lemma 2.4, it is convenient for us to use the continuous time approximation solution in our strong convergence analysis. Now, we define the two step functions for $t \in\left[t_{n}, t_{n+1}\right)$ as follows:

$$
\begin{aligned}
& Z_{1}(t)=Z_{1}(a, t)=\sum_{n=0}^{m-1} Q_{t}^{n} 1_{[n \Delta,(n+1) \Delta)}(t), \\
& Z_{2}(t)=Z_{2}(a, t)=\sum_{n=0}^{m-1} Q_{t}^{n *} 1_{[n \Delta,(n+1) \Delta)}(t),
\end{aligned}
$$

where $1_{G}$ is the indicator function for the set $G$. When $t \in\left[t_{n}, t_{n+1}\right)$, Lemma 2.4 can ensure the existence of $Q_{t}^{n *}$ for system (1). So we can define

$$
\begin{aligned}
Q_{t}= & Q_{t}^{n}+\left[-\frac{\partial Q_{t}^{n+1}}{\partial a}+(1-\theta)\left(-\mu(a, t) Q_{t}^{n}+f\left(t, Q_{t}^{n}\right)\right)\right]\left(t-t_{n}\right)+\theta\left(-\mu(a, t) Q_{t}^{n *}\right. \\
& \left.+f\left(t, Q_{t}^{n *}\right)\right)\left(t-t_{n}\right)+g\left(t, Q_{t}^{n *}\right)\left(B^{H}(t)-B^{H}\left(t_{n}\right)\right)+h\left(t, Q_{t}^{n *}\right)\left(N(t)-N\left(t_{n}\right)\right) .
\end{aligned}
$$

Thus the integral form of (6) can be written as follows:

$$
\begin{aligned}
Q_{t}= & K_{0}-\int_{0}^{t} \frac{\partial Q_{s}}{\partial a} d s+\int_{0}^{t}(1-\theta)\left[-\mu(a, s) Z_{1}(s)+f\left(s, Z_{1}(s)\right)\right] d s \\
& +\int_{0}^{t} \theta\left[-\mu(a, s) Z_{2}(s)+f\left(s, Z_{2}(s)\right)\right] d s+\int_{0}^{t} g\left(s, Z_{2}(s)\right) d B_{s}^{H} \\
& +\int_{0}^{t} h\left(s, Z_{s}(s)\right) d N_{s},
\end{aligned}
$$

with initial value $Q_{0}=K(a, 0), Q_{t}=K(a, t)$.

It is straightforward to check that $Z_{1}\left(a, t_{n}\right)=Q_{t}^{n}, Z_{2}\left(a, t_{n}\right)=Q_{t}^{n *}$, hence we regard $Q_{t}$ as a continuous-time extension of the discrete approximation $Q_{t}^{n}$, the main aim of this paper is to prove a strong convergence result for $Q_{t}$.

\section{Several lemmas}

In this section, we provide several lemmas which are useful for the proof of our results. 
The next two lemmas give the $p$ th moment boundedness of analytical solution $K_{t}$ and numerical solution $Q_{t}$ for (1). And the proofs of them are similar to those of Zhang et al. [21].

Lemma 3.1 Under assumptions (A1)-(A4), for any $p \geq 2$, there exists $C_{1}>0$ such that

$$
\mathbb{E}\left[\sup _{0 \leq t \leq T}\left|K_{t}\right|^{p}\right] \leq C_{1}
$$

Lemma 3.2 Under assumptions (A1)-(A4), for any $p \geq 2$, there exists a constant $C_{2}>0$ such that

$$
\mathbb{E}\left[\sup _{0 \leq t \leq T}\left|Q_{t}\right|^{p}\right] \leq C_{2}
$$

The next lemma shows the relationship between $\mathbb{E}\left|Q_{t}^{n *}\right|$ and $\mathbb{E}\left|Q_{t}^{n}\right|$.

Lemma 3.3 Under assumptions (A1)-(A4), let $\mathbb{E}\left|\frac{\partial Q_{t}^{n+1}}{\partial a}\right|^{2}<\infty$ and $0<\Delta<\min \left\{1, \frac{1}{\theta(l+\bar{\mu})}\right.$, $\left.\frac{1}{3 \sqrt{2\left(\bar{\mu}^{2}+l^{2}\right)}}\right\}$, there exist constants $C_{3}>0$ and $C_{4}>0$ such that

$$
\mathbb{E}\left|Q_{t}^{n *}\right|^{2} \leq C_{3}+C_{4} \mathbb{E}\left|Q_{t}^{n}\right|^{2}
$$

Proof Squaring both sides of (2) and using the elementary inequalities $(a+b+c)^{2} \leq 3 a^{2}+$ $3 b^{2}+3 c^{2}$ and $|(1-\theta) x+\theta y|^{2} \leq(1-\theta)|x|^{2}+\theta|y|^{2}$, we obtain

$$
\begin{aligned}
\left|Q_{t}^{n *}\right|^{2} \leq & 3\left|Q_{t}^{n}\right|^{2}+3\left|\frac{\partial Q_{t}^{n+1}}{\partial a}\right|^{2} \Delta^{2}+3(1-\theta) \Delta^{2}\left[-\mu\left(a, t_{n}\right) Q_{t}^{n}+f\left(t_{n}, Q_{t}^{n}\right)\right]^{2} \\
& +3 \theta \Delta^{2}\left[-\mu\left(a, t_{n}\right) Q_{t}^{n *}+f\left(t_{n}, Q_{t}^{n *}\right)\right]^{2} \\
\leq & 3\left|Q_{t}^{n}\right|^{2}+3\left|\frac{\partial Q_{t}^{n+1}}{\partial a}\right|^{2} \Delta^{2}+3 \Delta^{2}\left[\left(-\mu\left(a, t_{n}\right) Q_{t}^{n}+f\left(t_{n}, Q_{t}^{n}\right)\right)^{2}\right. \\
& \left.+\left(-\mu\left(a, t_{n}\right) Q_{t}^{n *}+f\left(t_{n}, Q_{t}^{n *}\right)\right)^{2}\right] .
\end{aligned}
$$

By assumptions (A2) and (A3), we get that

$$
\begin{aligned}
\left|Q_{t}^{n *}\right|^{2} \leq & 3\left|Q_{t}^{n}\right|^{2}+3\left|\frac{\partial Q_{t}^{n+1}}{\partial a}\right|^{2} \Delta^{2}+6 \Delta^{2}\left[\left|\mu\left(a, t_{n}\right) Q_{t}^{n}\right|^{2}+\left|f\left(t_{n}, Q_{t}^{n}\right)\right|^{2}\right] \\
& +6 \Delta^{2}\left[\left|\mu\left(a, t_{n}\right) Q_{t}^{n *}\right|^{2}+\left|f\left(t_{n}, Q_{t}^{n *}\right)\right|^{2}\right] \\
\leq & 3\left|Q_{t}^{n}\right|^{2}+3\left|\frac{\partial Q_{t}^{n+1}}{\partial a}\right|^{2} \Delta^{2}+6 \Delta^{2}\left(\bar{\mu}^{2}+l^{2}\right)\left|Q_{t}^{n}\right|^{2}+6 \Delta^{2}\left(\bar{\mu}^{2}+l^{2}\right)\left|Q_{t}^{n *}\right|^{2} .
\end{aligned}
$$

Taking mathematical expectation for both sides, we can get

$$
\mathbb{E}\left|Q_{t}^{n *}\right|^{2} \leq 3\left|\frac{\partial Q_{t}^{n+1}}{\partial a}\right|^{2} \Delta^{2}+3\left[1+2 \Delta^{2}\left(\bar{\mu}^{2}+l^{2}\right)\right] \mathbb{E}\left|Q_{t}^{n}\right|^{2}+6 \Delta^{2}\left(\bar{\mu}^{2}+l^{2}\right) \mathbb{E}\left|Q_{t}^{n *}\right|^{2} .
$$


Since $\Delta<\frac{1}{3 \sqrt{2\left(\bar{\mu}^{2}+l^{2}\right)}}$, thus $1-6 \Delta^{2}\left(\bar{\mu}^{2}+l^{2}\right) \geq \frac{1}{3}$ and $0<\Delta<1$, we have

$$
\begin{aligned}
\mathbb{E}\left|Q_{t}^{n *}\right|^{2} & \leq \frac{3 \mathbb{E}\left|\frac{\partial Q_{t}^{n+1}}{\partial a}\right|^{2} \Delta^{2}}{1-6 \Delta^{2}\left(\bar{\mu}^{2}+l^{2}\right)}+\frac{3\left[1+2 \Delta^{2}\left(\bar{\mu}^{2}+l^{2}\right)\right]}{1-6 \Delta^{2}\left(\bar{\mu}^{2}+l^{2}\right)} \mathbb{E}\left|Q_{t}^{n}\right|^{2} \\
& \leq 9 \mathbb{E}\left|\frac{\partial Q_{t}^{n+1}}{\partial a}\right|^{2}+9\left[1+2\left(\bar{\mu}^{2}+l^{2}\right)\right] \mathbb{E}\left|Q_{t}^{n}\right|^{2} \\
& :=C_{3}+C_{4} \mathbb{E}\left|Q_{t}^{n}\right|^{2},
\end{aligned}
$$

where $C_{3}=9 \mathbb{E}\left|\frac{\partial Q_{t}^{n+1}}{\partial a}\right|^{2}, C_{4}=9\left[1+2\left(\bar{\mu}^{2}+l^{2}\right)\right]$.

Lemma 3.4 Under assumptions (A1)-(A4), there exists a constant $C_{5}>0$ when $0<\Delta<$ $\min \left\{1, \frac{1}{\theta(l+\bar{\mu})}, \frac{1}{3 \sqrt{2\left(\bar{\mu}^{2}+l^{2}\right)}}\right\}$ such that

$$
\mathbb{E}\left[\sup _{0 \leq t \leq T}\left|Q_{t \wedge v_{n}}\right|^{2}\right] \leq C_{5}
$$

where $\tau_{n}=\inf \left\{t \geq 0,\left|K_{t}\right| \geq n\right\}, \sigma_{n}=\inf \left\{t \geq 0,\left|Q_{t}\right| \geq n\right\}$ are the first time that $K_{t}$ and $Q_{t}$ are unbounded respectively, and $v_{n}=\tau_{n} \wedge \sigma_{n}$.

Proof In terms of Eq. (7), we can obtain

$$
\begin{aligned}
d Q_{t}= & -\frac{\partial Q_{t}}{\partial a} d t+(1-\theta)\left[-\mu(a, t) Z_{1}(t)+f\left(t, Z_{1}(t)\right)\right] d t \\
& +\theta\left[-\mu(a, t) Z_{2}(t)+f\left(t, Z_{2}(t)\right)\right] d t \\
& +g\left(t, Z_{2}(t)\right) d B^{H}(t)+h\left(t, Z_{2}(t)\right) d N(t) .
\end{aligned}
$$

Applying Itô's formula to $\left|Q_{t \wedge v_{n}}\right|^{2}$ yields

$$
\begin{aligned}
\left|Q_{t \wedge v_{n}}\right|^{2}= & \left|Q_{0}\right|^{2}+2 \int_{0}^{t \wedge v_{n}}\left\langle-\frac{\partial Q_{s}}{\partial a}, Q_{s}\right\rangle d s-2 \int_{0}^{t \wedge v_{n}}\left((1-\theta) \mu(a, s) Z_{1}(s)\right. \\
& \left.+\theta \mu(a, s) Z_{2}(s), Q_{s}\right) d s+2 \int_{0}^{t \wedge v_{n}}\left((1-\theta) f\left(s, Z_{1}(s)\right)+\theta f\left(s, Z_{2}(s)\right), Q_{s}\right) d s \\
& +2 \int_{0}^{t \wedge v_{n}}\left(g\left(s, Z_{2}(s)\right), Q_{s}\right) d B^{H}(s)+2 \int_{0}^{t \wedge v_{n}}\left(h\left(s, Z_{2}(s)\right), Q_{s}\right) d N(s) \\
& +2 H \int_{0}^{t \wedge v_{n}} s^{2 H-1}\left\|g\left(s, Z_{2}(s)\right)\right\|_{2}^{2} d s+\int_{0}^{t \wedge v_{n}}\left|h\left(s, Z_{2}(s)\right)\right|^{2} d N(s) \\
\leq & \left|Q_{0}\right|^{2}+2 \int_{0}^{t \wedge v_{n}}\left\langle-\frac{\partial Q_{s}}{\partial a}, Q_{s}\right\rangle d s-2 \int_{0}^{t \wedge v_{n}}\left((1-\theta) \mu(a, s) Z_{1}(s)\right. \\
& \left.+\theta \mu(a, s) Z_{2}(s), Q_{s}\right) d s+2 \int_{0}^{t \wedge v_{n}}\left((1-\theta) f\left(s, Z_{1}(s)\right)+\theta f\left(s, Z_{2}(s)\right), Q_{s}\right) d s \\
& +2 \int_{0}^{t \wedge v_{n}}\left(g\left(s, Z_{2}(s)\right), Q_{s}\right) d B^{H}(s)+2 \int_{0}^{t \wedge v_{n}}\left(h\left(s, Z_{2}(s)\right), Q_{s}\right) d \bar{N}(s)
\end{aligned}
$$


Kang and Zhang Advances in Difference Equations (2018) 2018:371

Page 8 of 20

$$
\begin{aligned}
& +2 \lambda \int_{0}^{t \wedge v_{n}}\left(h\left(s, Z_{2}(s)\right), Q_{s}\right) d s+2 H \int_{0}^{t \wedge v_{n}} s^{2 H-1}\left\|g\left(s, Z_{2}(s)\right)\right\|_{2}^{2} d s \\
& +\int_{0}^{t \wedge v_{n}}\left|h\left(s, Z_{2}(s)\right)\right|^{2} d \bar{N}(s)+\lambda \int_{0}^{t \wedge v_{n}}\left|h\left(s, Z_{2}(s)\right)\right|^{2} d s,
\end{aligned}
$$

where $\bar{N}(s)=N(s)-\lambda s$ is a compensated Poisson process. By assumptions (A1)-(A3), we have

$$
\begin{aligned}
\left|Q_{t \wedge v_{n}}\right|^{2} \leq & \left|Q_{0}\right|^{2}+2 \int_{0}^{t \wedge v_{n}}\left\langle-\frac{\partial Q_{s}}{\partial a}, Q_{s}\right\rangle d s+2 \mu_{0} \int_{0}^{t \wedge v_{n}}\left|(1-\theta) Z_{1}(s)+\theta Z_{2}(s)\right|\left|Q_{s}\right| d s \\
& +2 \int_{0}^{t \wedge v_{n}}\left|(1-\theta) f\left(s, Z_{1}(s)\right)+\theta f\left(s, Z_{2}(s)\right)\right|\left|Q_{s}\right| d s \\
& +2 \int_{0}^{t \wedge v_{n}}\left(g\left(s, Z_{2}(s)\right), Q_{s}\right) d B^{H}(s)+2 \int_{0}^{t \wedge v_{n}}\left(h\left(s, Z_{2}(s)\right), Q_{s}\right) d \bar{N}(s) \\
& +2 \lambda \int_{0}^{t \wedge v_{n}}\left|h\left(s, Z_{2}(s)\right)\right|\left|Q_{s}\right| d s+2 H \int_{0}^{t \wedge v_{n}} s^{2 H-1}\left\|g\left(s, Z_{2}(s)\right)\right\|_{2}^{2} d s \\
& +\int_{0}^{t \wedge v_{n}}\left|h\left(s, Z_{2}(s)\right)\right|^{2} d \bar{N}(s)+\lambda \int_{0}^{t \wedge v_{n}}\left|h\left(s, Z_{2}(s)\right)\right|^{2} d s .
\end{aligned}
$$

Since

$$
\begin{aligned}
\left\langle-\frac{\partial Q_{s}}{\partial a}, Q_{s}\right\rangle & =-\int_{0}^{A} \frac{\partial Q_{s}}{\partial a} \cdot Q_{s} d a \\
& =\frac{1}{2} \gamma^{2}(s) A^{2}(s)\left[F\left(L(s), \int_{0}^{A} Q_{s} d a\right)-F(L(s), 0)\right]^{2} \\
& \leq \frac{1}{2} \eta^{2}\left(\left.\frac{\partial F(L, N)}{\partial N}\right|_{y}\right)^{2}\left(\int_{0}^{A} Q_{s} d a\right)^{2} \leq \frac{1}{2} A \eta^{2} \delta^{2}\left|Q_{s}\right|^{2},
\end{aligned}
$$

where $y \in\left(0, \int_{0}^{A} Q_{s} d a\right)$.

$$
\begin{aligned}
& 2 \mu_{0} \int_{0}^{t \wedge v_{n}}\left|(1-\theta) Z_{1}(s)+\theta Z_{2}(s)\right|\left|Q_{s}\right| d s \\
& \leq 2 \mu_{0} \int_{0}^{t \wedge v_{n}}\left(\left|Z_{1}(s)\right|^{2}+\left|Z_{2}(s)\right|^{2}\right) d s+\mu_{0} \int_{0}^{t \wedge v_{n}}\left|Q_{s}\right|^{2} d s, \\
& 2 \int_{0}^{t \wedge v_{n}}\left|(1-\theta) f\left(s, Z_{1}(s)\right)+\theta f\left(s, Z_{2}(s)\right)\right|\left|Q_{s}\right| d s \\
& \leq \int_{0}^{t \wedge v_{n}}\left|(1-\theta) f\left(s, Z_{1}(s)\right)+\theta f\left(s, Z_{2}(s)\right)\right|^{2} d s+\int_{0}^{t \wedge v_{n}}\left|Q_{s}\right|^{2} d s \\
& \leq 2 l^{2} \int_{0}^{t \wedge v_{n}}\left(\left|Z_{1}(s)\right|^{2}+\left|Z_{2}(s)\right|^{2}\right) d s+\int_{0}^{t \wedge v_{n}}\left|Q_{s}\right|^{2} d s, \\
& 2 \lambda \int_{0}^{t \wedge v_{n}}\left|h\left(s, Z_{2}(s)\right)\right|\left|Q_{s}\right| d s \leq \lambda \int_{0}^{t \wedge v_{n}}\left|h\left(s, Z_{2}(s)\right)\right|^{2} d s+\lambda \int_{0}^{t \wedge v_{n}}\left|Q_{s}\right|^{2} d s \\
& \quad \leq \lambda l^{2} \int_{0}^{t \wedge v_{n}}\left|Z_{2}(s)\right|^{2} d s+\lambda \int_{0}^{t \wedge v_{n}}\left|Q_{s}\right|^{2} d s .
\end{aligned}
$$


Taking (15)-(18) into (14), we get that

$$
\begin{aligned}
\left|Q_{t \wedge v_{n}}\right|^{2} \leq & \left|Q_{0}\right|^{2}+\left(A \eta^{2} \delta^{2}+\mu_{0}+1+\lambda\right) \int_{0}^{t \wedge v_{n}}\left|Q_{s}\right|^{2} d s \\
& +\left(2 \mu_{0}+2 l^{2}\right) \int_{0}^{t \wedge v_{n}}\left|Z_{1}(s)\right|^{2} d s \\
& +2\left[\mu_{0}+l^{2}\left(1+\lambda+H T^{2 H-1}\right)\right] \int_{0}^{t \wedge v_{n}}\left|Z_{2}(s)\right|^{2} d s \\
& +2 \int_{0}^{t \wedge v_{n}}\left(g\left(s, Z_{2}(s)\right), Q_{s}\right) d B^{H}(s) \\
& +2 \int_{0}^{t \wedge v_{n}}\left(h\left(s, Z_{2}(s)\right), Q_{s}\right) d \bar{N}(s)+\int_{0}^{t \wedge v_{n}}\left|h\left(s, Z_{2}(s)\right)\right|^{2} d \bar{N}(s),
\end{aligned}
$$

let $l_{1}=A \eta^{2} \delta^{2}+\mu_{0}+1+\lambda, l_{2}=2 \mu_{0}+2 l^{2}, l_{3}=2\left[\mu_{0}+l^{2}\left(1+\lambda+H T^{2 H-1}\right)\right]$, and $Z_{1}(s)=Q_{s}$, $Z_{2}(s)=Q_{s}^{*}$, and taking mathematical expectation for both sides of (19), we obtain

$$
\begin{aligned}
\mathbb{E}\left[\sup _{0 \leq s \leq t}\left|Q_{s \wedge v_{n}}\right|^{2}\right] \leq & \mathbb{E}\left|Q_{0}\right|^{2}+\left(l_{1}+l_{2}\right) \int_{0}^{t \wedge v_{n}} \mathbb{E}\left[\sup _{0 \leq s \leq t}\left|Q_{s}\right|^{2}\right] d s \\
& +l_{3} \int_{0}^{t \wedge v_{n}} \mathbb{E}\left[\sup _{0 \leq s \leq t}\left|Q_{s}^{*}\right|^{2}\right] d s \\
& +2 \mathbb{E}\left[\sup _{0 \leq s \leq t} \int_{0}^{t \wedge v_{n}}\left(g\left(s, Z_{2}(s)\right), Q_{s}\right) d B^{H}(s)\right] \\
& +2 \mathbb{E}\left[\sup _{0 \leq s \leq t} \int_{0}^{t \wedge v_{n}}\left(h\left(s, Z_{2}(s)\right), Q_{s}\right) d \bar{N}(s)\right] \\
& +\mathbb{E}\left[\sup _{0 \leq s \leq t} \int_{0}^{t \wedge v_{n}}\left|h\left(s, Z_{2}(s)\right)\right|^{2} d \bar{N}(s)\right] .
\end{aligned}
$$

Furthermore, by Lemma 3.3, we can get

$$
\begin{aligned}
\mathbb{E}\left[\sup _{0 \leq s \leq t}\left|Q_{s \wedge v_{n}}\right|^{2}\right] \leq & \mathbb{E}\left|Q_{0}\right|^{2}+\left(l_{1}+l_{2}+l_{3} C_{4}\right) \int_{0}^{t \wedge v_{n}} \mathbb{E}\left[\sup _{0 \leq s \leq t}\left|Q_{s}\right|^{2}\right] d s+l_{3} C_{3} T \\
& +2 \mathbb{E}\left[\sup _{0 \leq s \leq t} \int_{0}^{t \wedge v_{n}}\left(g\left(s, Z_{2}(s)\right), Q_{s}\right) d B^{H}(s)\right] \\
& +2 \mathbb{E}\left[\sup _{0 \leq s \leq t} \int_{0}^{t \wedge v_{n}}\left(h\left(s, Z_{2}(s)\right), Q_{s}\right) d \bar{N}(s)\right] \\
& +\mathbb{E}\left[\sup _{0 \leq s \leq t} \int_{0}^{t \wedge v_{n}}\left|h\left(s, Z_{2}(s)\right)\right|^{2} d \bar{N}(s)\right]
\end{aligned}
$$

By the Burkholder-Davis-Gundy inequality, we have

$$
\begin{aligned}
2 \mathbb{E} & {\left[\sup _{0 \leq s \leq t} \int_{0}^{t \wedge v_{n}}\left(g\left(s, Z_{2}(s)\right), Q_{s}\right) d B^{H}(s)\right] } \\
& \leq C \mathbb{E}\left[\sup _{0 \leq s \leq t}\left|Q_{s \wedge v_{n}}\right|\left(\int_{0}^{t \wedge v_{n}}\left\|g\left(s, Z_{2}(s)\right)\right\|_{2}^{2} B^{H}(s)\right)^{\frac{1}{2}}\right]
\end{aligned}
$$




$$
\begin{aligned}
& \leq \frac{1}{6} \mathbb{E}\left[\sup _{0 \leq s \leq t}\left|Q_{s \wedge v_{n}}\right|^{2}\right]+l_{1}^{\prime} \int_{0}^{t \wedge v_{n}} s^{2 H-1} \mathbb{E}\left\|g\left(s, Z_{2}(s)\right)\right\|_{2}^{2} d s \\
& \leq \frac{1}{6} \mathbb{E}\left[\sup _{0 \leq s \leq t}\left|Q_{s \wedge v_{n}}\right|^{2}\right]+l_{1}^{\prime} l^{2} C_{4} T^{2 H-1} \int_{0}^{t \wedge v_{n}} \mathbb{E}\left|Q_{s}\right|^{2} d s+l_{1}^{\prime} l^{2} C_{3} T^{2 H} .
\end{aligned}
$$

In the same way, we can get

$$
\begin{aligned}
2 \mathbb{E} & {\left[\sup _{0 \leq s \leq t} \int_{0}^{t \wedge v_{n}}\left(h\left(s, Z_{2}(s)\right), Q_{s}\right) d \bar{N}(s)\right] } \\
& \leq \frac{1}{6} \mathbb{E}\left[\sup _{0 \leq s \leq t}\left|Q_{s \wedge v_{n}}\right|^{2}\right]+l_{2}^{\prime} l^{2} C_{4} \int_{0}^{t \wedge v_{n}} \mathbb{E}\left|Q_{s}\right|^{2} d s+l_{2}^{\prime} l^{2} C_{3} T,
\end{aligned}
$$

and

$$
\begin{aligned}
& \mathbb{E}\left[\sup _{0 \leq s \leq t} \int_{0}^{t \wedge v_{n}}\left|h\left(s, Z_{2}(s)\right)\right|^{2} d \bar{N}(s)\right] \\
& \quad \leq \frac{1}{6} \mathbb{E}\left[\sup _{0 \leq s \leq t}\left|Q_{s \wedge v_{n}}\right|^{2}\right]+l_{3}^{\prime} l^{2} C_{4} \int_{0}^{t \wedge v_{n}} \mathbb{E}|Q(s)|^{2} d s+l_{3}^{\prime} l^{2} C_{3} T+C_{3},
\end{aligned}
$$

where $l_{1}^{\prime}, l_{2}^{\prime}, l_{3}^{\prime}>0$. Substituting (22)-(24) into (21) and denoting $l^{\prime}=\max \left\{l_{1}^{\prime}, l_{2}^{\prime}, l_{3}^{\prime}\right\}$, we have

$$
\begin{aligned}
\mathbb{E}\left[\sup _{0 \leq s \leq t}\left|Q_{s \wedge v_{n}}\right|^{2}\right] \leq & 2 \mathbb{E}\left|Q_{0}\right|^{2}+2 l^{\prime} l^{2} C_{3} T\left(2+T^{2 H-1}\right)+2 C_{3}+2 l_{3} C_{3} T \\
& +2\left[l_{1}+l_{2}+l_{3} C_{4}+l^{\prime} l^{2} C_{4}\left(2+T^{2 H-1}\right)\right] \int_{0}^{t \wedge v_{n}} \mathbb{E}\left[\sup _{0 \leq s \leq t}\left|Q_{s}\right|^{2}\right] d s \\
:= & l_{4}+l_{5} \int_{0}^{t \wedge v_{n}} \mathbb{E}\left[\sup _{0 \leq s \leq t}\left|Q_{s}\right|^{2}\right] d s
\end{aligned}
$$

where

$$
\begin{aligned}
& l_{4}=2 \mathbb{E}\left|Q_{0}\right|^{2}+2 l^{\prime} l^{2} C_{3} T\left(2+T^{2 H-1}\right)+2 C_{3}+2 l_{3} C_{3} T, \\
& l_{5}=2\left[l_{1}+l_{2}+l_{3} C_{4}+l^{\prime} l^{2} C_{4}\left(2+T^{2 H-1}\right)\right] .
\end{aligned}
$$

Now, using the Gronwall lemma, we can easily obtain the following result:

$$
\mathbb{E}\left[\sup _{0 \leq s \leq t}\left|Q_{s \wedge v_{n}}\right|^{2}\right] \leq C_{5}
$$

where $C_{5}=l_{4} e^{l_{5} T}$. For $\forall t \in[0, T]$, we easily get

$$
\mathbb{E}\left[\sup _{0 \leq t \leq T}\left|Q_{t \wedge v_{n}}\right|^{2}\right] \leq C_{5}
$$

This completes the proof.

Lemma 3.5 Under assumptions (A1)-(A4), let $0<\Delta<\min \left\{1, \frac{1}{\theta(l+\bar{\mu})}, \frac{1}{3 \sqrt{2\left(\bar{\mu}^{2}+l^{2}\right)}}\right\}$ for any $t \in[n \Delta,(n+1) \Delta) \subseteq[0, T]$, and for some positive integer $n(n=0,1, \ldots)$, there exist constants 
$C_{6}>0, C_{7}>0$, which are independent of $\Delta$, such that

$$
\begin{aligned}
& \mathbb{E}\left|Q_{t}-Z_{1}(t)\right|^{2} \leq C_{6} \Delta, \\
& \mathbb{E}\left|Q_{t}-Z_{2}(t)\right|^{2} \leq C_{7} \Delta .
\end{aligned}
$$

Proof Considering $t \in[n \Delta,(n+1) \Delta) \subseteq[0, T]$ and according to (7), we have

$$
\begin{aligned}
Q_{t}-Z_{1}(t)= & Q_{t}-Q_{t}^{n} \\
= & \int_{n \Delta}^{t} \frac{\partial Q_{s}}{\partial a} d s+\int_{n \Delta}^{t}\left[(1-\theta) f\left(s, Z_{1}(s)\right)+\theta f\left(s, Z_{2}(s)\right)\right] d s \\
& -\int_{n \Delta}^{t} \mu(a, s)\left[(1-\theta) Z_{1}(s)+\theta Z_{2}(s)\right] d s+\int_{n \Delta}^{t} g\left(s, Z_{2}(s)\right) d B^{H}(s) \\
& +\int_{n \Delta}^{t} h\left(s, Z_{2}(s)\right) d N(s) .
\end{aligned}
$$

Squaring both sides of Eq. (28), using the elementary inequality $\left(\sum_{i=1}^{n} x_{i}\right)^{2} \leq n \sum_{i=1}^{n} x_{i}^{2}$, the Cauchy-Schwarz inequality, and (A3), we have

$$
\begin{aligned}
\left|Q_{t}-Z_{1}(t)\right|^{2} \leq & 5\left|\int_{n \Delta}^{t} \frac{\partial Q_{s}}{\partial a} d s\right|^{2}+5\left|\int_{n \Delta}^{t}\left[(1-\theta) f\left(s, Z_{1}(s)\right)+\theta f\left(s, Z_{2}(s)\right)\right] d s\right|^{2} \\
& +5\left|\int_{n \Delta}^{t} \mu(a, s)\left[(1-\theta) Z_{1}(s)+\theta Z_{2}(s)\right] d s\right|^{2}+5\left|\int_{n \Delta}^{t} g\left(s, Z_{2}(s)\right) d B^{H}(s)\right|^{2} \\
& +5\left|\int_{n \Delta}^{t} h\left(s, Z_{2}(s)\right) d N(s)\right|^{2} \\
\leq & 5 \Delta \int_{n \Delta}^{t}\left|\frac{\partial Q_{s}}{\partial a}\right|^{2} d s+5 \Delta \int_{n \Delta}^{t}\left|(1-\theta) f\left(s, Z_{1}(s)\right)+\theta f\left(s, Z_{2}(s)\right)\right|^{2} d s \\
& +5 \bar{\mu}^{2} \Delta \int_{n \Delta}^{t}\left|(1-\theta) Z_{1}(s)+\theta Z_{2}(s)\right|^{2} d s+5\left|\int_{n \Delta}^{t} g\left(s, Z_{2}(s)\right) d B^{H}(s)\right|^{2} \\
& +10\left|\int_{n \Delta}^{t} h\left(s, Z_{2}(s)\right) d \bar{N}(s)\right|^{2}+10 \lambda^{2}\left|\int_{n \Delta}^{t} h\left(s, Z_{2}(s)\right) d s\right|^{2}
\end{aligned}
$$

where $\bar{N}(s)=N(s)-\lambda s$ is a compensated Poisson process. Taking mathematical expectation and by assumptions (A1)-(A4), we have

$$
\begin{aligned}
\mathbb{E}\left|Q_{t}-Z_{1}(t)\right|^{2} \leq & 5 \Delta \int_{n \Delta}^{t}\left|\frac{\partial Q_{s}}{\partial a}\right|^{2} d s+10 \Delta \int_{n \Delta}^{t}\left[\mathbb{E}\left|f\left(s, Z_{1}(s)\right)\right|^{2}+\mathbb{E}\left|f\left(s, Z_{2}(s)\right)\right|^{2}\right] d s \\
& +10 \bar{\mu}^{2} \Delta \int_{n \Delta}^{t}\left[\mathbb{E}\left|Z_{1}(s)\right|^{2}+\mathbb{E}\left|Z_{2}(s)\right|^{2}\right] d s \\
& +10 H \int_{n \Delta}^{t} s^{2 H-1} \mathbb{E}\left\|g\left(s, Z_{2}(s)\right)\right\|^{2} d s \\
& +10 \lambda \int_{n \Delta}^{t} \mathbb{E}\left|h\left(s, Z_{2}(s)\right)\right|^{2} d s+10 \lambda^{2} \Delta \int_{n \Delta}^{t} \mathbb{E}\left|h\left(s, Z_{2}(s)\right)\right|^{2} d s
\end{aligned}
$$




$$
\begin{aligned}
\leq & 5 \Delta \int_{n \Delta}^{t}\left|\frac{\partial Q_{s}}{\partial a}\right|^{2} d s+10 \Delta\left(l^{2}+\bar{\mu}^{2}\right) \int_{n \Delta}^{t} \mathbb{E}\left|Z_{1}(s)\right|^{2} d s \\
& +10\left[\Delta\left(l^{2}+\bar{\mu}^{2}\right)+l^{2}\left(H T^{2 H-1}+\lambda+\lambda^{2} \Delta\right)\right] \int_{n \Delta}^{t} \mathbb{E}\left|Z_{2}(s)\right|^{2} d s .
\end{aligned}
$$

Since, for all $t \in[n \Delta,(n+1) \Delta)$, we have $Z_{1}(t)=Q_{t}^{n}, Z_{2}(t)=Q_{t}^{n *}$, by Lemmas 3.3 and 3.4, we get

$$
\begin{aligned}
\mathbb{E}\left|Q_{t}-Z_{1}(t)\right|^{2} \leq & 5 \Delta \int_{n \Delta}^{t}\left|\frac{\partial Q_{s}}{\partial a}\right|^{2} d s+10\left[\Delta\left(l^{2}+\bar{\mu}^{2}\right)+l^{2}\left(H T^{2 H-1}+\lambda+\lambda^{2} \Delta\right)\right] C_{3} \Delta \\
& +10\left[\Delta\left(l^{2}+\bar{\mu}^{2}\right)+C_{4}\left(\Delta\left(l^{2}+\bar{\mu}^{2}\right)+l^{2}\left(H T^{2 H-1}+\lambda+\lambda^{2} \Delta\right)\right)\right] C_{2} \Delta \\
:= & C_{6} \Delta,
\end{aligned}
$$

where $C_{6}=5 \int_{n \Delta}^{t}\left|\frac{\partial Q_{s}}{\partial a}\right|^{2} d s+10 C_{3}\left[\Delta\left(l^{2}+\bar{\mu}^{2}\right)+l^{2}\left(H T^{2 H-1}+\lambda+\lambda^{2} \Delta\right)\right]+10 C_{2}\left[\Delta\left(l^{2}+\bar{\mu}^{2}\right)+\right.$ $\left.C_{4}\left(\Delta\left(l^{2}+\bar{\mu}^{2}\right)+l^{2}\left(H T^{2 H-1}+\lambda+\lambda^{2} \Delta\right)\right)\right]$. Thus, we have proved (26), similarly, we can prove (27).

Remark 3.6 Lemma 3.5 indicates that the continuous-time approximation $Q_{t}$ in (7) can arbitrarily close to the step functions $Z_{1}(t)$ and $Z_{2}(t)$ in the mean square sense when $\Delta \rightarrow 0$.

\section{The main results and proofs}

In this section, we give the main results of this paper and provide the detailed proofs by using the lemmas in the previous section.

Theorem 4.1 Under assumptions (A1)-(A4), let $0<\Delta<\min \left\{1, \frac{1}{\theta(l+\bar{\mu})}, \frac{1}{3 \sqrt{2\left(\bar{\mu}^{2}+l^{2}\right)}}\right\}$ for all $t \in[n \Delta,(n+1) \Delta) \subseteq[0, T]$, there exists a constant $C_{8}>0$ such that

$$
\mathbb{E}\left[\sup _{0 \leq t \leq T}\left|K_{t \wedge v_{n}}-Q_{t \wedge v_{n}}\right|^{2}\right] \leq C_{8} \Delta
$$

Proof The integral version of the first equation in system (1) is given as follows:

$$
\begin{aligned}
K_{t}= & K_{0}-\int_{0}^{t} \frac{\partial K_{s^{-}}}{\partial a} d s-\int_{0}^{t} \mu(a, s) K_{s^{-}} d s+\int_{0}^{t} f\left(s, K_{s^{-}}\right) d s \\
& +\int_{0}^{t} g\left(s, K_{s^{-}}\right) d B^{H}(s)+\int_{0}^{t} h\left(s, K_{s^{-}}\right) d N(s) .
\end{aligned}
$$

Combining (31) and (7), we can get

$$
\begin{aligned}
K_{t}-Q_{t}= & -\int_{0}^{t} \frac{\partial\left(K_{s}-Q_{s}\right)}{\partial a} d s-\int_{0}^{t} \mu(a, s)\left[(1-\theta)\left(K_{s}-Z_{1}(s)\right)+\theta\left(K_{s}-Z_{2}(s)\right] d s\right. \\
& +\int_{0}^{t}\left[(1-\theta)\left(f\left(s, K_{s}\right)-f\left(s, Z_{1}(s)\right)\right)+\theta\left(f\left(s, K_{s}\right)-f\left(s, Z_{2}(s)\right)\right)\right] d s \\
& +\int_{0}^{t}\left[g\left(s, K_{s}\right)-g\left(s, Z_{2}(s)\right)\right] d B^{H}(s)+\int_{0}^{t}\left[h\left(s, K_{s}\right)-h\left(s, Z_{2}(s)\right)\right] d N(s) .
\end{aligned}
$$


Applying Itô’s formula to $\left|K_{t}-Q_{t}\right|^{2}$, for $\forall t \in[0, T]$, yields

$$
\begin{aligned}
\left|K_{t}-Q_{t}\right|^{2}= & 2 \int_{0}^{t}\left\langle-\frac{\partial\left(K_{s}-Q_{s}\right)}{\partial a}, K_{s}-Q_{s}\right\rangle d s \\
- & 2 \int_{0}^{t}\left(\mu(a, s)\left[(1-\theta)\left(K_{s}-Z_{1}(s)\right)+\theta\left(K_{s}-Z_{2}(s)\right)\right], K_{s}-Q_{s}\right) d s \\
& +2 \int_{0}^{t}\left((1-\theta)\left(f\left(s, K_{s}\right)-f\left(s, Z_{1}(s)\right)\right)+\theta\left(f\left(s, K_{s}\right)-f\left(s, Z_{2}(s)\right)\right), K_{s}-Q_{s}\right) d s \\
& +2 \int_{0}^{t}\left(g\left(s, K_{s}\right)-g\left(s, Z_{2}(s)\right), K_{s}-Q_{s}\right) d B^{H}(s) \\
& +2 H \int_{0}^{t} s^{2 H-1}\left\|g\left(s, K_{s}\right)-g\left(s, Z_{2}(s)\right)\right\|_{2}^{2} d s \\
& +2 \int_{0}^{t}\left(h\left(s, K_{s}\right)-h\left(s, Z_{2}(s)\right), K_{s}-Q_{s}\right) d \bar{N}(s) \\
& +\int_{0}^{t}\left|h\left(s, K_{s}\right)-h\left(s, Z_{2}(s)\right)\right|^{2} d \bar{N}(s) \\
& +2 \lambda \int_{0}^{t}\left(h\left(s, K_{s}\right)-h\left(s, Z_{2}(s)\right), K_{s}-Q_{s}\right) d s+\lambda \int_{0}^{t} \mid h\left(s, K_{s}\right)-h\left(s,\left.Z_{2}(s)\right|^{2} d s .\right.
\end{aligned}
$$

Using the Cauchy-Schwarz inequality and (A2)-(A4), we have

$$
\begin{aligned}
\left|K_{t}-Q_{t}\right|^{2} \leq & A \eta^{2} \delta^{2} \int_{0}^{t}\left|K_{s}-Q_{s}\right|^{2} d s \\
& +2 \mu_{0} \int_{0}^{t}\left|K_{s}-Q_{s}\right| \mid(1-\theta)\left(K_{s}-Z_{1}(s)\right)+\theta\left(K_{s}-Z_{2}(s) \mid d s\right. \\
& +2 \int_{0}^{t}\left|K_{s}-Q_{s}\right|\left|(1-\theta)\left(f\left(s, K_{s}\right)-f\left(s, Z_{1}(s)\right)\right)+\theta\left(f\left(s, K_{s}\right)-f\left(s, Z_{2}(s)\right)\right)\right| d s \\
& +2 \int_{0}^{t}\left(g\left(s, K_{s}\right)-g\left(s, Z_{2}(s)\right), K_{s}-Q_{s}\right) d B^{H}(s) \\
& +2 H \int_{0}^{t} s^{2 H-1}|| g\left(s, K_{s}\right)-g\left(s, Z_{2}(s)\right) \|_{2}^{2} d s \\
& +2 \int_{0}^{t}\left(h\left(s, K_{s}\right)-h\left(s, Z_{2}(s)\right), K_{s}-Q_{s}\right) d \bar{N}(s) \\
& +\int_{0}^{t}\left|h\left(s, K_{s}\right)-h\left(s, Z_{2}(s)\right)\right|^{2} d \bar{N}(s) \\
& +2 \lambda \int_{0}^{t}\left|K_{s}-Q_{s}\right|\left|h\left(s, K_{s}\right)-h\left(s, Z_{2}(s)\right)\right| d s \\
& +\lambda \int_{0}^{t}\left|h\left(s, K_{s}\right)-h\left(s, Z_{2}(s)\right)\right|^{2} d s \\
\leq & \left(A \eta^{2} \delta^{2}+\mu_{0}+1+\lambda\right) \int_{0}^{t}\left|K_{s}-Q_{s}\right|^{2} d s+2\left(\mu_{0}+l^{2}\right) \int_{0}^{t}\left|K_{s}-Z_{1}(s)\right|^{2} d s \\
& +2\left[\mu_{0}+l^{2}\left(1+\lambda+H T^{2 H-1}\right)\right] \int_{0}^{t}\left|K_{s}-Z_{2}(s)\right|^{2} d s
\end{aligned}
$$




$$
\begin{aligned}
& +2 \int_{0}^{t}\left(g\left(s, K_{s}\right)-g\left(s, Z_{2}(s)\right), K_{s}-Q_{s}\right) d B^{H}(s) \\
& +2 \int_{0}^{t}\left(h\left(s, K_{s}\right)-h\left(s, Z_{2}(s)\right), K_{s}-Q_{s}\right) d \bar{N}(s) \\
& +\int_{0}^{t}\left|h\left(s, K_{s}\right)-h\left(s, Z_{2}(s)\right)\right|^{2} d \bar{N}(s) \\
& =l_{1} \int_{0}^{t}\left|K_{s}-Q_{s}\right|^{2} d s+l_{2} \int_{0}^{t}\left|K_{s}-Z_{1}(s)\right|^{2} d s+l_{3} \int_{0}^{t}\left|K_{s}-Z_{2}(s)\right|^{2} d s \\
& +2 \int_{0}^{t}\left(g\left(s, K_{s}\right)-g\left(s, Z_{2}(s)\right), K_{s}-Q_{s}\right) d B^{H}(s) \\
& +\int_{0}^{t}\left|h\left(s, K_{s}\right)-h\left(s, Z_{2}(s)\right)\right|^{2} d \bar{N}(s) \\
& +2 \int_{0}^{t}\left(h\left(s, K_{s}\right)-h\left(s, Z_{2}(s)\right), K_{s}-Q_{s}\right) d \bar{N}(s) .
\end{aligned}
$$

Hence, for any $t \in[0, T]$, we have

$$
\begin{aligned}
\mathbb{E}\left[\sup _{0 \leq s \leq t}\left|K_{s \wedge v_{n}}-Q_{s \wedge v_{n}}\right|^{2}\right] \\
\leq l_{1} \int_{0}^{s \wedge v_{n}} \mathbb{E} \sup _{0 \leq r \leq s}\left|K_{r \wedge v_{n}}-Q_{r \wedge v_{n}}\right|^{2} d r \\
\quad+l_{2} \int_{0}^{s \wedge v_{n}} \mathbb{E} \sup _{0 \leq r \leq s}\left|K_{r}-Z_{1}(r)\right|^{2} d r+l_{3} \int_{0}^{s \wedge v_{n}} \mathbb{E} \sup _{0 \leq r \leq s}\left|K_{r}-Z_{2}(r)\right|^{2} d r \\
\quad+2 \mathbb{E} \sup _{0 \leq s \leq t} \int_{0}^{s \wedge v_{n}}\left(g\left(r, K_{r}\right)-g\left(r, Z_{2}(r)\right), K_{r}-Q_{r}\right) d B^{H}(r) \\
\quad+2 \mathbb{E} \sup _{0 \leq s \leq t} \int_{0}^{s \wedge v_{n}}\left(h\left(r, K_{r}\right)-h\left(r, Z_{2}(r)\right), K_{r}-Q_{r}\right) d \bar{N}(r) \\
\quad+\mathbb{E} \sup _{0 \leq s \leq t}^{s \wedge v_{n}}\left|h\left(r, K_{r}\right)-h\left(r, Z_{2}(r)\right)\right|^{2} d \bar{N}(r) .
\end{aligned}
$$

By the Burkholder-Davis-Gundy inequality and Lemma 3.5, we get

$$
\begin{aligned}
2 \mathbb{E} & \sup _{0 \leq s \leq t} \int_{0}^{s \wedge v_{n}}\left(g\left(r, K_{r}\right)-g\left(r, Z_{2}(r)\right), K_{r}-Q_{r}\right) d B^{H}(r) \\
\leq & C \mathbb{E}\left[\sup _{0 \leq s \leq t}\left|K_{s \wedge v_{n}}-Q_{s \wedge v_{n}}\right|\left(\int_{0}^{s \wedge v_{n}}\left\|g\left(r, K_{r}\right)-g\left(r, Z_{2}(r)\right)\right\|_{2}^{2} d B^{H}(r)\right)^{\frac{1}{2}}\right] \\
\leq & \frac{1}{6} \mathbb{E}\left[\sup _{0 \leq s \leq t}\left|K_{s \wedge v_{n}}-Q_{s \wedge v_{n}}\right|^{2}\right]+l_{6} \int_{0}^{s \wedge v_{n}} r^{2 H-1} \mathbb{E}\left|K_{r}-Z_{2}(r)\right|^{2} d r \\
\leq & \frac{1}{6} \mathbb{E}\left[\sup _{0 \leq s \leq t}\left|K_{s \wedge v_{n}}-Q_{s \wedge v_{n}}\right|^{2}\right] \\
& +2 l_{6} T^{2 H-1} \int_{0}^{s \wedge v_{n}} \mathbb{E}\left|K_{r}-Q_{r}\right|^{2} d r+2 l_{6} C_{7} T^{2 H-1} \Delta .
\end{aligned}
$$


In the same way, we have

$$
\begin{aligned}
& 2 \mathbb{E} \sup _{0 \leq s \leq t} \int_{0}^{s \wedge v_{n}}\left(h\left(r, K_{r}\right)-h\left(r, Z_{2}(r)\right), K_{r}-Q_{r}\right) d \bar{N}(r) \\
& \leq \frac{1}{6} \mathbb{E}\left[\sup _{0 \leq s \leq t}\left|K_{s \wedge v_{n}}-Q_{s \wedge v_{n}}\right|^{2}\right]+2 l_{7} \int_{0}^{s \wedge v_{n}} \mathbb{E}\left|K_{r}-Q_{r}\right|^{2} d r+2 l_{7} C_{7} \Delta \\
& \mathbb{E} \sup _{0 \leq s \leq t} \int_{0}^{s \wedge v_{n}}\left|h\left(r, K_{r}\right)-h\left(r, Z_{2}(r)\right)\right|^{2} d \bar{N}(r) \\
& \leq C \mathbb{E}\left[\sup _{0 \leq s \leq t} \int_{0}^{s \wedge v_{n}}\left|h\left(r, K_{r}\right)-h\left(r, Z_{2}(r)\right)\right|^{4} d r\right]^{\frac{1}{2}} \\
& \leq \frac{1}{6} \mathbb{E}\left[\sup _{0 \leq s \leq t}\left|K_{s \wedge v_{n}}-Z_{2}(s)\right|^{2}\right]+l_{8} \int_{0}^{s \wedge v_{n}} \mathbb{E}\left|K_{r}-Z_{2}(r)\right|^{2} d r \\
& \leq \frac{1}{3} \mathbb{E}\left[\sup _{0 \leq s \leq t}\left|K_{s \wedge v_{n}}-Q_{s \wedge v_{n}}\right|^{2}\right]+\frac{1}{3} \mathbb{E}\left[\sup _{0 \leq s \leq t}\left|Q_{s \wedge v_{n}}-Z_{2}(s)\right|^{2}\right] \\
&+2 l_{8} \int_{0}^{s \wedge v_{n}} \mathbb{E}\left|K_{r}-Q_{r}\right|^{2} d r+2 l_{8} C_{7} \Delta,
\end{aligned}
$$

where $l_{6}, l_{7}, l_{8}>0$. Let $l^{\prime \prime}=\max \left\{l_{6}, l_{7}, l_{8}\right\}$, inserting (34)-(36) into (33), we obtain

$$
\begin{aligned}
\mathbb{E}\left[\sup _{0 \leq s \leq t}\left|K_{s \wedge v_{n}}-Q_{s \wedge v_{n}}\right|^{2}\right] \\
\leq l_{1} \int_{0}^{s \wedge v_{n}} \mathbb{E} \sup _{0 \leq r \leq s}\left|K_{r \wedge v_{n}}-Q_{r \wedge v_{n}}\right|^{2} d r \\
\quad+2\left[l_{2}+l_{3}+l^{\prime \prime}\left(T^{2 H-1}+2\right)\right] \int_{0}^{s \wedge v_{n}} \mathbb{E}\left|K_{r}-Q_{r}\right|^{2} d r \\
\quad+\frac{2}{3} \mathbb{E}\left[\sup _{0 \leq s \leq t}\left|K_{s \wedge v_{n}}-Q_{s \wedge v_{n}}\right|^{2}\right]+2\left[l_{2} C_{6}+\left(l_{3}+3 l^{\prime \prime}+\frac{1}{6} C_{7}\right)\right] \Delta,
\end{aligned}
$$

i.e.,

$$
\begin{aligned}
\mathbb{E}\left[\sup _{0 \leq s \leq t}\left|K_{s \wedge v_{n}}-Q_{s \wedge v_{n}}\right|^{2}\right] \\
\leq 3\left[l_{1}+2\left(l_{2}+l_{3}+l^{\prime \prime}\left(T^{2 H-1}+2\right)\right)\right] \int_{0}^{s \wedge v_{n}} \mathbb{E} \sup _{0 \leq r \leq s}\left|K_{r \wedge v_{n}}-Q_{r \wedge v_{n}}\right|^{2} d r \\
\quad+6\left[l_{2} C_{6}+\left(l_{3}+l^{\prime \prime} T^{2 H-1}+2 l^{\prime \prime}+\frac{1}{6}\right) C_{7}\right] \Delta \\
:=D_{1} \Delta+D_{2} \int_{0}^{s \wedge v_{n}} \mathbb{E} \sup _{0 \leq r \leq s}\left|K_{r \wedge v_{n}}-Q_{r \wedge v_{n}}\right|^{2} d r,
\end{aligned}
$$

where $D_{1}=6\left[l_{2} C_{6}+\left(l_{3}+l^{\prime \prime} T^{2 H-1}+2 l^{\prime \prime}+\frac{1}{6}\right) C_{7}\right], D_{2}=3\left[l_{1}+2\left(l_{2}+l_{3}+l^{\prime \prime}\left(T^{2 H-1}+2\right)\right)\right]$. Using the Gronwall inequality, we have

$$
\mathbb{E}\left[\sup _{0 \leq s \leq t}\left|K_{s \wedge v_{n}}-Q_{s \wedge v_{n}}\right|^{2}\right] \leq D_{1} \exp \left\{D_{2} T\right\} \Delta
$$


where $C_{8}=D_{1} \exp \left\{D_{2} T\right\}$. Hence, for $\forall t \in[0, T]$, we have

$$
\mathbb{E}\left[\sup _{0 \leq s \leq t}\left|K_{s \wedge v_{n}}-Q_{s \wedge v_{n}}\right|^{2}\right] \leq C_{8} \Delta .
$$

This completes the proof.

Theorem 4.2 Under assumptions (A1)-(A4), let $0<\Delta<\min \left\{1, \frac{1}{\theta(l+\bar{\mu})}, \frac{1}{3 \sqrt{2\left(\bar{\mu}^{2}+l^{2}\right)}}\right\}$, then there exists a constant $C_{9}>0$ such that

$$
\mathbb{E}\left[\sup _{0 \leq t \leq T}\left|K_{t}-Q_{t}\right|^{2}\right] \leq C_{9} \Delta
$$

Proof Let $e_{t}=K_{t}-Q_{t}$, we can directly obtain

$$
\begin{aligned}
\mathbb{E}\left[\sup _{0 \leq t \leq T}\left|e_{t}\right|^{2}\right] & =\mathbb{E}\left[\sup _{0 \leq t \leq T}\left|e_{t}\right|^{2} 1_{\left\{\tau_{n}>T \text { and } \sigma_{n}>T\right\}}\right]+\mathbb{E}\left[\sup _{0 \leq t \leq T}\left|e_{t}\right|^{2} 1_{\left\{\tau_{n} \leq T \text { or } \sigma_{n} \leq T\right\}}\right] \\
& =\mathbb{E}\left[\sup _{0 \leq t \leq T}\left|e_{t}\right|^{2} 1_{\left\{v_{n}>T\right\}}\right]+\mathbb{E}\left[\sup _{0 \leq t \leq T}\left|e_{t}\right|^{2} 1_{\left\{\tau_{n} \leq T \text { or } \sigma_{n} \leq T\right\}}\right] \\
& \leq \mathbb{E}\left[\sup _{0 \leq t \leq T}\left|e_{t \wedge v_{n}}\right|^{2}\right]+\mathbb{E}\left[\sup _{0 \leq t \leq T}\left|e_{t}\right|^{2} 1_{\left\{\tau_{n} \leq T \text { or } \sigma_{n} \leq T\right\}}\right] .
\end{aligned}
$$

Applying the Young inequality $x y \leq \frac{1}{p} x^{p}+\frac{1}{q} y^{q}\left(\frac{1}{p}+\frac{1}{q}=1, p, q>0\right)$, we have

$$
\mathbb{E}\left[\sup _{0 \leq t \leq T}\left|e_{t}\right|^{2} 1_{\left\{\tau_{n} \leq T \text { or } \sigma_{n} \leq T\right\}}\right] \leq \frac{\Delta}{2} \mathbb{E}\left[\sup _{0 \leq t \leq T}\left|e_{t}\right|^{4}\right]+\frac{1}{2 \Delta} P\left\{\tau_{n} \leq T \text { or } \sigma_{n} \leq T\right\} .
$$

Obviously,

$$
\mathbb{E}\left[\sup _{0 \leq t \leq T}\left|e_{t}\right|^{4}\right] \leq 8\left(\mathbb{E}\left[\sup _{0 \leq t \leq T}\left|K_{t}\right|^{4}\right]+\mathbb{E}\left[\sup _{0 \leq t \leq T}\left|Q_{t}\right|^{4}\right]\right) \leq 8\left(C_{1}+C_{2}\right) .
$$

On the other hand,

$$
P\left\{\tau_{n} \leq T\right\}=\mathbb{E}\left[1_{\left\{\tau_{n} \leq T\right\}} \frac{\left|K_{\tau_{n}}\right|^{4}}{n^{4}}\right]=\frac{1}{n^{4}} \mathbb{E}\left[\sup _{0 \leq t \leq T}\left|K_{t}\right|^{4}\right] \leq \frac{C_{1}}{n^{4}}
$$

and

$$
P\left\{\sigma_{n} \leq T\right\}=\mathbb{E}\left[1_{\left\{\sigma_{n} \leq T\right\}} \frac{\left|Q_{\tau_{n}}\right|^{4}}{n^{4}}\right]=\frac{1}{n^{4}} \mathbb{E}\left[\sup _{0 \leq t \leq T}\left|Q_{t}\right|^{4}\right] \leq \frac{C_{2}}{n^{4}} .
$$

So,

$$
P\left\{\tau_{n} \leq T \text { or } \sigma_{n} \leq T\right\} \leq P\left\{\tau_{n} \leq T\right\}+P\left\{\sigma_{n} \leq T\right\} \leq \frac{C_{1}+C_{2}}{n^{4}} .
$$

Substituting (43) and (44) into (42), we get

$$
\mathbb{E}\left[\sup _{0 \leq t \leq T}\left|e_{t}\right|^{2} 1_{\left\{\tau_{n} \leq T \text { or } \sigma_{n} \leq T\right\}}\right] \leq 4 \Delta\left(C_{1}+C_{2}\right)+\frac{1}{2 \Delta n^{4}}\left(C_{1}+C_{2}\right) .
$$


By Theorem 4.1, (41) can be written

$$
\mathbb{E}\left[\sup _{0 \leq t \leq T}\left|e_{t}\right|^{2}\right] \leq C_{8} \Delta+4 \Delta\left(C_{1}+C_{2}\right)+\frac{1}{2 \Delta n^{4}}\left(C_{1}+C_{2}\right)+o(\Delta) .
$$

Let $n \geq\left(24 \Delta^{2}\right)^{-\frac{1}{4}}$, we get that

$$
\begin{aligned}
\mathbb{E}\left[\sup _{0 \leq t \leq T}\left|e_{t}\right|^{2}\right] & \leq C_{8} \Delta+4 \Delta\left(C_{1}+C_{2}\right)+2 \Delta\left(C_{1}+C_{2}\right) \\
& =\Delta\left[C_{8}+6\left(C_{1}+C_{2}\right)\right]:=C_{9} \Delta,
\end{aligned}
$$

where $C_{9}=C_{8}+6\left(C_{1}+C_{2}\right)$.

Letting $\Delta \rightarrow 0$, yields

$$
0 \leq \mathbb{E}\left[\sup _{0 \leq t \leq T}\left|K_{t}-Q_{t}\right|^{2}\right] \leq 0,
$$

which means

$$
\mathbb{E}\left[\sup _{0 \leq t \leq T}\left|K_{t}-Q_{t}\right|^{2}\right]=0
$$

Thus, we can obtain the following theorem.

Theorem 4.3 Underassumptions (A1)-(A4), let $0<\Delta<\min \left\{1, \frac{1}{\theta(l+\bar{\mu})}, \frac{1}{3 \sqrt{2\left(\bar{\mu}^{2}+l^{2}\right)}}\right\}$, then the numerical solution will converge to the analytic solution to system (1) in the mean square sense

$$
\lim _{\Delta \rightarrow 0} \mathbb{E}\left[\sup _{0 \leq t \leq T}\left|K_{t}-Q_{t}\right|^{2}\right]=0
$$

Proof The result of this theorem can be directly got by Theorem 4.2.

\section{Remark 4.4}

(1) Theorem 4.2 shows that the value of $\theta$ has an effect on step size $\Delta$, and it further affects the convergence of solution.

(2) Theorem 4.3 implies that the numerical solution and the analytic solution to system (1) can arbitrarily close when step size $\Delta \rightarrow 0$.

\section{Numerical experiments}

In this section, we present an example to verify our theoretical results. Let us consider the following stochastic age-dependent capital system with Poisson jumps and fBm:

$$
\begin{cases}\frac{\partial K(a, t)}{\partial t}=\left[-\frac{\partial K_{t^{-}}}{\partial a}-\frac{1}{1-a} K_{t^{-}}\right] d t+K_{t^{-}} d B^{H}(t)-K_{t^{-}} d N(t), & (a, t) \in(0, A) \times(0, T), \\ K(0, t)=\int_{0}^{1} \frac{1}{(1-a)^{2}} K(a, t) d a, & t \in[0, T], \\ K(a, 0)=\exp (1 /(a-1)), & a \in[0, A], \\ N(t)=\int_{0}^{1} K(a, t) d a, & t \in[0, T],\end{cases}
$$


where $B^{H}(t)$ is a fBm with Hurst parameter $H=\frac{3}{4}$, and $N(t)$ is a scalar Poisson process with intensity $\lambda=1$. Take $A=1, T=1$ in (48), and $W=L^{2}([0,1]), V=H^{1}([0,1])$ (a Sobolev space with elements satisfying the boundary conditions above), the depreciation rate $\mu(a, t)=$ $\frac{1}{1-a}, \gamma(t) A(t)=1$, the production function $F(L(t), N(t))=2 \int_{0}^{1} K(a, t) d a$, the labor force $L(t)=2, f(t, K)=0, g(t, K)=K$, and $h(t, K)=-K$.

It is easy to verify that operators $f, g$, and $h$ satisfy conditions (A1)-(A4). Then the approximate solution will converge to the true solution of Eq. (48) for any $(a, t) \in(0,1) \times(0,1)$ according to Theorem 4.3. Figures 1 and 2 are numerical simulations of the stochastic cap-
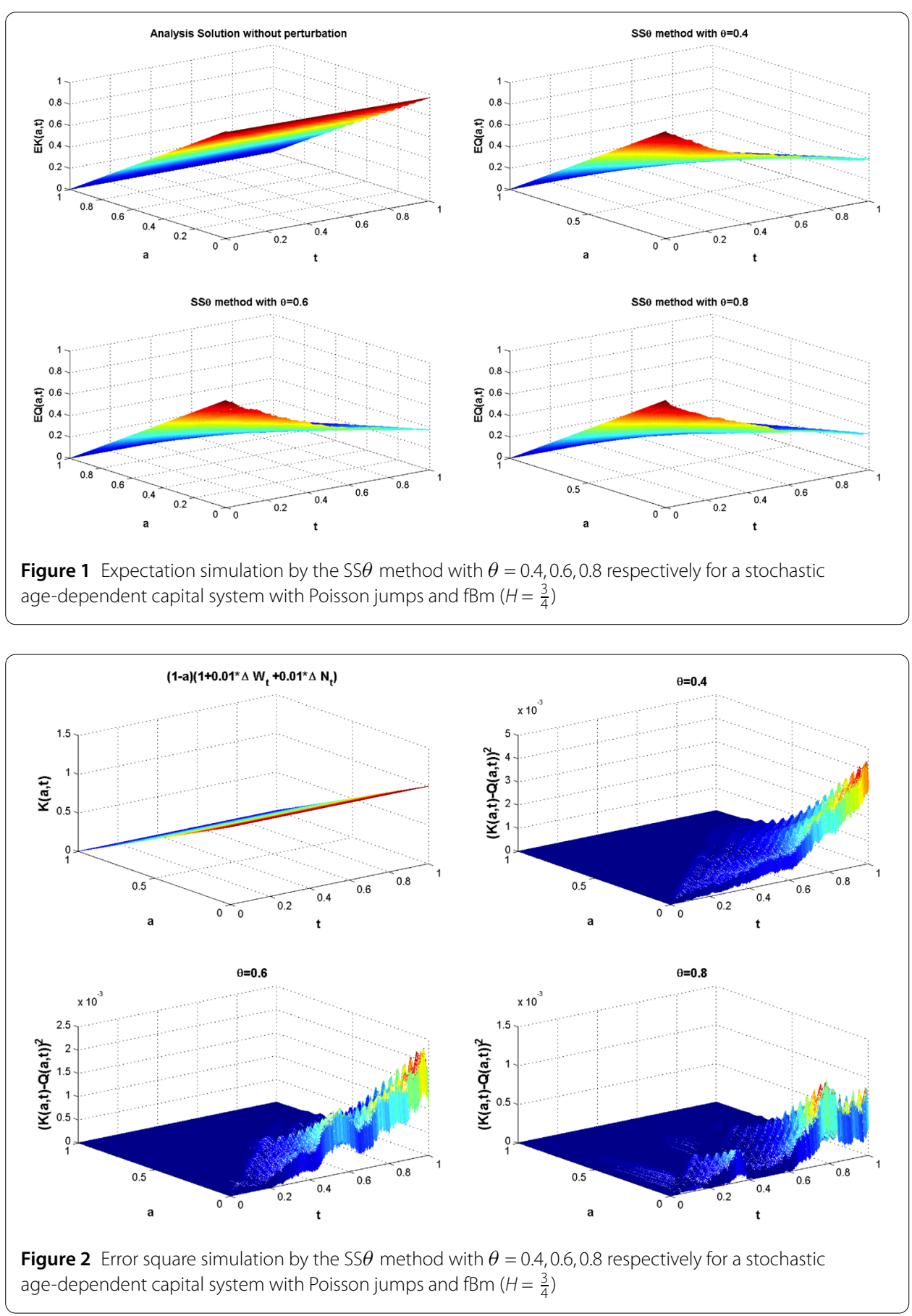
ital system with Poisson jumps and fractional Brownian motion ( $\mathrm{fBm}$ ) when $\Delta t=0.001$, $\Delta a=0.01$, and $K(a, t)=E Q(a, t)=1 / 1000 \sum_{k=1}^{1000} Q_{k}(a, t)$. It is difficult to obtain the true explicit solution to (48), so the analysis solution $K(a, t)$ to Eq. (48) can be replaced by $(1+a)\left(1+0.01 \Delta B_{t}^{H}+0.01 \Delta N_{t}\right)$.

In Fig. 1, we plot the analysis solution and numerical solution of (48) with $\theta=0.4,0.6,0.8$ respectively. The analysis solution of Eq. (48) without perturbation is $E K(a, t)=1-a$, and we can easily find that the numerical approximation will tend to the analysis solution in the mean sense.

In Fig. 2, we show the error square of analysis solution and numerical solution obtained from the $\operatorname{SS} \theta$ method with $\theta=0.4,0.6,0.8$ respectively. It is easy to verify that the maximum value of the error square for the $\operatorname{SS} \theta$ approximation is not greater than 0.01 , which is smaller than the error of the EM method [3] and the SSBE method [4].

\title{
6 Conclusion
}

The uncertainty factors in the financial market are usually fluctuating, discontinuous, and recurrent, and these important factors contain technological progress, productivity of new products, natural disasters, changes in laws and government policies, and so on. As we all known, the aforementioned factors have essential effects on the profitability of risky assets. In order to resolve this problem, we introduce a class of stochastic age-dependent capital systems with Poisson jumps and $\mathrm{fBm}$ in this paper. Nevertheless, most stochastic age-dependent capital models are nonlinear and cannot be solved explicitly, so appropriate numerical approximation schemes are needed to study the properties of models. In this paper, we have established some new results on the convergence of the split-step $\theta$ method for system (1) and proved that the numerical approximation solutions converge to the analytic solutions of the equations under the given conditions. Meanwhile, the order of approximation is also provided. Finally, an example has effectively demonstrated our theoretical results.

There are still many interesting issues to be studied in future. For example, how to select the value of $\theta$ to make the error small? Whether we can apply other new numerical methods to system (1) and obtain similar results? We leave these problems for future investigations.

\author{
Acknowledgements \\ The authors are grateful to two anonymous referees for several pertinent questions and suggestions. \\ Funding \\ The research was supported by the Natural Science Foundation of China (11661064), Ningxia College Scientific Research \\ Project (NGY2017217), and the Natural Science Foundation of Ningxia University (ZR16002). \\ Competing interests \\ The authors declare that they have no competing interests. \\ Authors' contributions \\ All authors contributed equally to this work. All authors read and approved the final manuscript. \\ Author details \\ ${ }^{1}$ School of Mathematics and Statistics, Ningxia University, Yinchuan, P.R. China. ${ }^{2}$ Xinhua College, Ningxia University, \\ Yinchuan, P.R. China.
}

Publisher's Note

Springer Nature remains neutral with regard to jurisdictional claims in published maps and institutional affiliations.

Received: 8 May 2018 Accepted: 1 October 2018 Published online: 11 October 2018 


\section{References}

1. Zhang, Q., Pang, W., Leung, P.: Exponential stability of numerical solutions for a class of stochastic age-dependent capital system with Poisson jumps. J. Comput. Appl. Math. 235(12), 3369-3377 (2011)

2. Zhang, Q.: Convergence of numerical solutions for a class of stochastic age-dependent capital system with Markovian switching. Econ. Model. 28(3), 1195-1201 (2011)

3. Zhang, Q., Rathinasamy, A.: Convergence of numerical solutions for a class of stochastic age-dependent capital system with random jump magnitudes. Appl. Math. Comput. 219(14), 7297-7305 (2013)

4. Zhang, Q., Liu, Y., Li, X.: Strong convergence of split-step backward Euler method for stochastic age-dependent capital system with Markovian switching. Appl. Math. Comput. 235, 439-453 (2014)

5. Gu, H., Liang, J., Zhang, Y.: Time-changed geometric fractional Brownian motion and option pricing with transaction costs. Phys. A, Stat. Mech. Appl. 391(15), 3971-3977 (2012)

6. Katarzyna, J.-B.: Generalized BSDEs driven by fractional Brownian motion. Stat. Probab. Lett. 83(3), 805-811 (2013)

7. Jiang, Y., Wang, X., Wang, Y.: On a stochastic heat equation with first order fractional noises and applications to finance. J. Math. Anal. Appl. 396(2), 656-669 (2012)

8. Wang, J., Liang, J., Lv, L., Qiu, W., Ren, F.: Continuous time Black-Scholes equation with transaction costs in subdiffusive fractional Brownian motion regime. Phys. A, Stat. Mech. Appl. 391(3), 750-759 (2012)

9. Li, Q., Kang, T., Zhang, Q.: Mean-square dissipative methods for stochastic age-dependent capital system with fractional Brownian motion and jumps. Appl. Math. Comput. 339, 81-92 (2018)

10. Ding, X., Ma, Q., Zhang, L.: Convergence and stability of the split-step $\theta$-method for stochastic differential equations. Comput. Math. Appl. 60(5), 1310-1321 (2010)

11. Rathinasamy, A., Balachandran, K.: T-stability of the split-step $\theta$-methods for linear stochastic delay integro-differential equations. Nonlinear Anal. Hybrid Syst. 5(4), 639-646 (2011)

12. Rathinasamy, A.: The split-step $\theta$-methods for stochastic delay Hopfield neural networks. Appl. Math. Model. 36(8), 3477-3485 (2012)

13. Rathinasamy, A.: Split-step $\theta$-methods for stochastic age-dependent population equations with Markovian switching. Nonlinear Anal., Real World Appl. 13(3), 1334-1345 (2012)

14. Cao, W., Hao, P., Zhang, Z.: Split-step $\theta$-method for stochastic delay differential equations. Appl. Numer. Math. 76, 19-33 (2014)

15. Huang, C.: Mean square stability and dissipativity of two classes of theta methods for systems of stochastic delay differential equations. J. Comput. Appl. Math. 259, 77-86 (2014)

16. Li, X., Cao, W.: On mean-square stability of two-step Maruyama methods for nonlinear neutral stochastic delay differential equations. Appl. Math. Comput. 261, 373-381 (2015)

17. Tan, J., Rathinasamy, A., Pei, Y.: Convergence of the split-step $\theta$-method for stochastic age-dependent population equations with Poisson jumps. Appl. Math. Comput. 254, 305-317 (2015)

18. Zong, X., Wu, F., Xu, G.: Convergence and stability of two classes of theta-Milstein schemes for stochastic differential equations. J. Comput. Appl. Math. 336, 8-29 (2018)

19. Wang, X., Gan, S.: The improved split-step backward Euler method for stochastic differential delay equations. Int. J. Comput. Math. 88(11), 2359-2378 (2011)

20. Tan, J., Men, W., Pei, Y., Guo, Y.: Construction of positivity preserving numerical method for stochastic age-dependent population equations. Appl. Math. Comput. 293, 57-64 (2017)

21. Zhang, Q., Liu, W., Nie, Z:: Existence, uniqueness and exponential stability for stochastic age-dependent population. Appl. Math. Comput. 154(1), 183-201 (2004)

22. Smart, D.: Fixed Point Theorems. Cambridge University Press, Cambridge (1974)

\section{Submit your manuscript to a SpringerOpen ${ }^{\circ}$ journal and benefit from:}

- Convenient online submission

- Rigorous peer review

- Open access: articles freely available online

- High visibility within the field

- Retaining the copyright to your article

Submit your next manuscript at $\gg$ springeropen.com 